\title{
A Novel T-CAD Framework to Support Medical Image Analysis and Reconstruction
}

\author{
Danilo Avola ${ }^{1}$, Luigi Cinque ${ }^{1}$, and Marco Di Girolamo ${ }^{2}$ \\ 1 Sapienza University of Rome, Department of Computer Science \\ Via Salaria 113, 00198 Rome, Italy \\ \{avola, cinque\}@di.uniroma1.it \\ http://w3.uniroma1.it/dipinfo/english/index.asp \\ 2 Sapienza University of Rome, S.Andrea Hospital, Department of Diagnostic Science \\ Via di Grottarossa 1035-1039, 00189 Rome, Italy \\ digirolamomarco@hotmail.com \\ http://www.ospedalesantandrea.it/index.php
}

\begin{abstract}
The current medical imaging devices allow to obtain high resolution digital images with a complex informative content expressed by the textural skin that covers organs and tissues (hereinafter objects). These textural information can be exploited to develop a descriptive mathematical model of the objects by which to support heterogeneous activities within the medical field.

This paper describes our developed framework based on the texture analysis by which to mathematically model every object contained in the layout of the total body NMR images. By every specific model, the framework automatically also defines a connected application which supports, on the related object, different fixed targets, such as: segmentation, mass detection, reconstruction, and so on.
\end{abstract}

Keywords: Framework, CAD, medical image, texture analysis, pattern recognition, feature extraction, segmentation, classification.

\section{Introduction}

The current medical imaging devices (e.g. Nuclear Magnetic Resonance (NMR), Positron Emission Tomography (PET)) allow to obtain digital images with high level details. These images have a complex informative content that goes beyond the simple visual representation. More specifically, by observing the relationships between clusters of pixels (i.e. the texture) of the skin that covers the objects can be brought out the meaningful features by which to describe the morphological structures related to objects themselves. These features (i.e. textural information) are exploited to develop a mathematical model, of the objects, able to support different activities within the medical field. In the last years, there have been many efforts to conceive intelligent and automated systems to support several critical medical tasks (e.g. analysis, masses identification). The making of the current Decision Support Systems (DSSs), better known in this area as Computer Aided Diagnosis (CAD) systems, are still not very effective tools. 
This paper describes a generalized texture analysis and reconstruction approach, based on our previous experiences shown in [1], [2] and [3], by which to support the total body NMR images. In particular, the paper details our developed Texture based Computer Aided Diagnosis Framework (T-CAD Framework) which, on one side, supports the mathematical modeling process of each organ and tissue represented inside the total body NMR image layout and, on the other hand, automatically defines, by every specific model, an ad-hoc application by which to support a set of fixed targets useful to aid the medical specialist in a specific context (e.g. craniopharyngioma identification on NMR encephalic images). Observe that every mathematical model (e.g. craniopharyngioma model) achieved during the modeling process is defined one and for all.

There is an extensive literature focused on the different aspects of the medical image processing. These approaches are based on several principles related to the image understanding, but there are no works able to exploit the morphological structure (given by the texture analysis) of the objects with the aim to provide both their descriptive mathematical model and related dedicated T-CAD system. The effectiveness of the proposed approach is shown both experimental results and the ad-hoc model-driven applications.

A first work that has driven some our choices in image analysis approach is shown in [4], where the authors describe an automatic segmentation framework, for brain Magnetic Resonance Imaging (MRI), based on the combination of atlas registration, fuzzy connectedness segmentation, and parametric bias field correction. Another work that has supported some aspects related to the textural image filters is detailed in [5], where the authors present a novel co-occurrence matrixes based approach able to discriminate the texture belonging to different kind of images by considering the statistical representation of the structural texture primitives (i.e. textons). Another useful approach is presented in [6], where the authors presents an approach to automate the myocardial contours detection in order to optimize the detection and the tracking of the grid of tags within myocardium. A further work that has supported our framework is shown in [7]. In this work the authors present a real mixed statistical model based on a region-driven curve evolution algorithm. An original approach that has assisted some aspects of our texture based image processing is described in [8], where the authors introduce a novel algorithm to achieve automatic texture based segmentation of organs in MRIs of the abdomen. An innovative approach, which has inspired some solutions of our textural model, is detailed in [9], where the authors present a robust multi-resolution statistical shape model algorithm. A last remarkable work that has aided our collaboration process between textural filters is presented in 10, where the authors explore the use of the co-occurrence matrixes to extract textural features from medical images.

The paper is organized as follows. Section 2 illustrates the main architectural aspects of the T-CAD Framework. Section 3 presents a concrete case study including the related mathematical model. Section 4 introduces and discusses the main experimental results. Section 5 concludes and plans the future work. 


\section{The T-CAD Framework Architecture}

The developed T-CAD Framework is a smart tool that allows skilled user to define a texture based Mathematical Model (MM) of every organ and tissue represented inside the NMR total body image layout. A specific MM (e.g. of the brain) is simply the set of formalized mathematical classes which represent the different basic objects contained in the related NMR image (e.g. cerebral tissue, abnormal mass, background). For every MM the framework supports the building of a dedicated T-CAD system to support a specific medical image analysis process (e.g. NMR encephalic image segmentation and mass recognition).

The next two sub-sections respectively show the general approach, and the main textural filters used within the framework.

\subsection{The Region Based Algorithm}

The Region Based Algorithm (RBA) can be considered the core of the whole T-CAD Framework. Actually, its main aspect regards the building of all the mathematical classes that made up a specific MM, inasmuch the dedicated system generation is only a technical application of the related MM on a dataset of source images. For this reason, the explanation will mainly detailed the definition of the MM, and finally will be highlighted the system generation process. Figure 1 shows the simplified architecture of the region based algorithm.

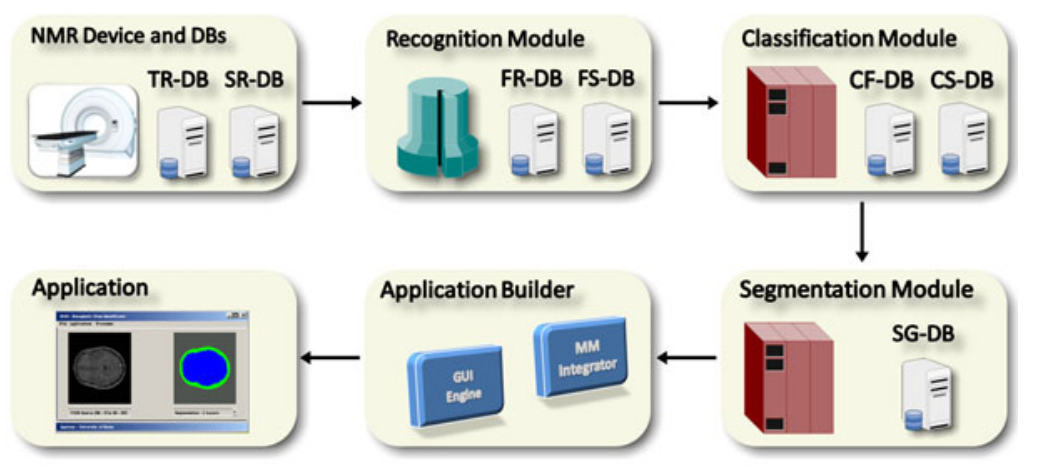

Fig. 1. Region Based Algorithm Architecture

The first panel (NMR Device and DBs) highlights that our framework works on two kind of DB. The first (TRaining-DB) is used when the skilled user has to build a new MM. For this reason, the populating of the TR-DB follows a rigid protocol which has to ensure different qualitative and quantitative requirements relatively to the informative content of the related images. The second DB (SouRce-DB) points out that, once obtained the related MM, it is possible to analyze every kind of source image coming directly form the NMR device. 
The second panel (Recognition Module) highlights the recognition process on every image belonging to the TR-DB, where an adaptable elaboration window runs across the image to perform a feature extraction process based on a suitable set of textural filters (i.e. features vector). The start step, supported by two specific filters: Entropy and Homogeneity (see next sub-section), is to fix the window size both maximizing the number of image zones with high entropy levels and minimizing the number of neighboring heterogeneous zones. The final step of the process is to exploit the found fixed window to analyze, by the features vector (which filters are stored in the FilteR-DB), every image area (in top-bottom and left-right way). For each image the analysis process produces a feature map, the set of feature maps defines a feature space (stored in the Feature SpaceDB), all of which, suitably interpreted, provide the mathematical class of every chosen basic object. In fact, by studying the correlation related to all the feature spaces of each basic object can be defined a preliminary mathematical model of everyone. Afterwards, the exhaustive comparison of each different preliminary model allows skilled user to find the textural relationships to univocally describe each basic object. This description is the MM, which can be considered as the set of the mathematical classes that define itself. Observe that the MM definition is a supervised process in which the final formalization of the numerical classes is still left to the human specialist. Moreover, the process only occurs when a skilled user wants to build a new MM.

The third panel (Classification Module) points out the classification process which exploits the MM found in the previous module (and stored in the ClassFormat-DB) with the aim to analyze the source images coming form the NMR device (SR-DB). This process follows the same approach shown in the recognition module, but its purpose is to classify any zone of every source image according to a selected MM. The module works following two different steps. During the first any image zone is analyzed (by the just mentioned elaboration window) to classify it according to one of the formalized mathematical classes belonging to the related MM. Afterwards, homogeneous image zones are suitably marked and merged. During the second step the module assigns to one of the formalized classes, by a statistical distribution algorithm, the possible image zones that have been not classified at all.

The fourth panel (Segmentation Module) highlights the segmentation process where every classified source image is properly labeled in order to to provide an immediate visual impact to the user. Every segmented image is arranged in a suitable DB (SeGmented-DB).

The fifth panel (Application Builder) points out the ad-hoc application builder. In particular, the MM Integrator will include the definition of the selected MM inside the application. Observe that the framework allows user to include more than one specific MM. The GUI Engine highlights that the main mechanisms related to the data presentation (e.g. visualization engine, interaction properties) are the same independently from the specific application.

The sixth panel (Application) shows an example of a created application which performs the encaphalic NMR image segmentation. 


\subsection{The Textural Image Filters}

The image filters adopted to support the RBA have been suitably chosen and/or created to define the basic textural informative content of the tissues and organs belonging to the human body. Actually, our strong belief is to have found a general approach adoptable for every object represented by the NMR images, which, at the moment, has been refined to define the textural morphological structures of four specific objects: brain, heart, liver and bony structure.

Our approach subdivide the image filters within three different graphical classes, each one able to characterize a specific informative layer of the mentioned objects: informative class, texture class and pattern class.

The informative class is made up by those first order statistic image filters which distinguish between zone with and without relevant information content. In our experience, the following two set of filters represent the main best suitable ones: $N$-Order Moment $\left(M_{n_{1}}\right)$ and $N$-Order Central Moment $\left(C_{n_{2}}\right)$ :

$$
M_{n_{1}}=\sum_{i=0}^{N} i^{n_{1}} \times p(i), C_{n_{2}}=\sum_{i=0}^{N}\left(i-M_{n_{1}}\right)^{n_{2}} \times p(i)
$$

Where: $p(i)$ represents the probability that the gray level value $i$ appears inside the elaboration window. The following constraints must be respected:

$$
0 \leq p(i) \leq 1 \forall i \in[0 . .255] \subset \mathbb{N}, \sum_{i=0}^{N} p(i)=1, n_{1}, n_{2} \in \mathbb{N}, N=255
$$

The $M_{n_{1}}$ and $C_{n_{2}}$ set of filters respectively measure, on different textural graphical layers, the consistent quantity and the semantic readability of the information related to different image zones.

The texture class is made up by those second order statistic image filters which measure the macro and micro textural structures. Our empirical studies have allowed to detect the following four set of filters: Homogeneity $(H g(d))$, Contrast $(C t(d))$, Inverse Difference $(\operatorname{Id}(d))$ and Entropy $(\operatorname{En}(d))$ :

$$
\begin{aligned}
& H g(d)=\sum_{i=0}^{N} \sum_{j=0}^{N}\left[p_{d}(i, j)\right]^{2}, C t(d)=\sum_{i=0}^{N} \sum_{j=0}^{N}|i, j|\left[p_{d}(i, j)\right]^{l} \\
& I d(d)=\sum_{i=0}^{N} \sum_{j=0}^{N} \frac{p_{d}(i, j)^{m}}{1+(i-j)^{k}}, \operatorname{En}(d)=-\sum_{i=0}^{N} \sum_{j=0}^{N} p_{d}(i, j) \log _{n}\left(p_{d}(i, j)\right)
\end{aligned}
$$

Where: $p_{d}(i, j)$ represents the probability that two points with distance $d$ have respectively $i$ and $j$ gray value. The following constraints must be respected:

$$
\begin{aligned}
& 0 \leq p_{d}(i, j) \leq 1, \forall(i, j) \in[0 . .255] \times[0 . .255] \subset \mathbb{N}^{2} \\
& \sum_{i=0}^{N} \sum_{j=0}^{N} p_{d}(i, j)=1, d \in[1 . .8] \subset \mathbb{N}, l, m, k, n \subset \mathbb{N}, N=255
\end{aligned}
$$

The $H g(d)$ set of filters measure the degree of uniformity of the different image zones, where high or low values within the feature maps respectively highlight light or wide changes of the textural structures. The $C t(d)$ set of filters express 
how roughly occur the mentioned structural changes, where high values, of the related feature maps, point out fast continuous changes within the image zones, on the contrary, they give low values. The $I d(d)$ set of filters provide the measure of the transition between different basic objects, where low values typically highlight a boundary zone. The $E n(d)$ set of filters is used to detect the randomness level within the considered image zone, where the complex changes in the random distribution of the grey levels are directly proportional to the given values.

The pattern class is made up by those second order statistic image filters which measure the pattern structures. Our experimental observations have supported to identify the following two set of filters: Correlation $(\mathrm{Cr}(d))$, Difference Entropy $(\mathrm{De}(d))$ :

$$
C r(d)=\sum_{i=0}^{N} \sum_{j=0}^{N} \frac{\left(i-\mu_{x}\right)\left(j-\mu_{y}\right) p_{d}(i, j)^{l}}{\left[\sigma_{x} \sigma_{y}\right]^{m}}, D e(d)=-\sum_{i=0}^{N} p_{x-y}(i) \log _{n}\left[p_{x-y}(i)\right]
$$

Where:

$$
\begin{aligned}
& \mu_{x}=\sum_{i=0}^{N} \sum_{j=0}^{N} i\left(p_{d}(i, j)\right), \sigma_{x}=\sqrt{\sum_{i=0}^{N} \sum_{j=0}^{N}\left(i-\mu_{x}\right)^{2}\left(p_{d}(i, j)\right)} \\
& \mu_{y}=\sum_{i=0}^{N} \sum_{j=0}^{N} j\left(p_{d}(i, j)\right), \sigma_{y}=\sqrt{\sum_{i=0}^{N} \sum_{j=0}^{N}\left(i-\mu_{y}\right)^{2}\left(p_{d}(i, j)\right)} \\
& p_{x-y}(k)=\sum_{i=0}^{N} \sum_{j=0}^{N}\left[p_{d}(i, j)\right]^{q}, \text { where }|i-j|=k
\end{aligned}
$$

The $C r(d)$ set of filters are usually used to recognize definite patterns within texture zones previously identified, while the $D e(d)$ set of filters is adopted to detect the different components (i.e. parts of a same pattern) of different basic objects.

Every class of image filters is based on our variation of the current co-occurrence matrix concept, where the elaboration process considers all the search directions. In particular, every couple of pixels, able to increase a coefficient of the matrix, is chosen considering a pixel (i.e. center of a circle) within the elaboration window and the connected pixel which is at the boundary of the circumference related to the fixed radius (i.e. the $d$ parameter).

All the parameters belonging to the set of filters are customized, within the recognition process, according to the specific contextual medical domain and related tasks. Besides, the shown filters are often used on more than one level of the Gaussian Pyramid ([1] ) by which to enrich the resolution of the texture described through the MMs.

\section{Case Study: Brain}

This section shows a concrete case study in which a suitable mathematical model has been defined according to specific targets, and where a model-based 
application has been created to support the related image analysis process. Actually, the framework essentially produces the same application in which only the mathematical model is replaced every time.

Observe that the morphological structures of organs and tissues are very different, each one of they can be better emphasized according to a specific kind of NMR image (e.g. $T_{1}, T_{2}$, proton density). For this reason, a brief specification of the DICOM (Digital Imaging and Communication in Medicine) image format is given in relation to a specific case study.

\subsection{NMR Encephalic Mass Identificator}

The Encephalic Mass Identification (EMI) application has been created with the aim to aid medical specialists during the mass identification within the encaphalic NMR images. In particular, the following targets were established:

a. segmentation of the image layout in three basic objects: cerebral tissue, rest of the image (i.e. muscular and bony structure) and background;

b. identification within the cerebral tissue of the abnormal masses (e.g. gliomas, craniopharyngiomas, medulloblastomas);

c. classification of the found abnormal masses as craniopharyngioma pathology distinguishing it from other kinds of primary cerebral tumors.

A careful analysis of the set of pixel that composes the encephalic NMR images has detected in the transversal $T_{1}$ weighted the more suitable ones to better highlight the textural morphological structures of the objects, according to the fixed targets. Table 1 shows the main technical features of the images.

Table 1. NMR Encephalic Images: Main Technical Features

\begin{tabular}{|c|c|c|c|c|c|c|c|}
\hline \multirow{2}{*}{$\begin{array}{c}\text { Main } \\
\begin{array}{c}\text { Technical } \\
\text { Features }\end{array}\end{array}$} & \multicolumn{2}{|c|}{ Resolution } & \multicolumn{2}{c|}{ Category } & \multicolumn{2}{|c|}{ Pre-Processing } & Scanning \\
\cline { 2 - 7 } & Spatial & Color & $\begin{array}{c}\text { Primary } \\
\text { Type }\end{array}$ & $\begin{array}{c}\text { Secondary } \\
\text { Type }\end{array}$ & $\begin{array}{c}\text { Primary } \\
\text { Type }\end{array}$ & $\begin{array}{c}\text { Secondary } \\
\text { Type }\end{array}$ & $\begin{array}{c}\text { Anatomic } \\
\text { Plane }\end{array}$ \\
\hline $\begin{array}{c}\text { NMR } \\
\text { Encephalic } \\
\text { Images }\end{array}$ & $512 \times 512$ & $256(8$ bit) & $\begin{array}{c}\mathrm{T}_{1} \\
\text { (weighted and } \\
\text { not weighted) }\end{array}$ & $\begin{array}{c}\mathrm{T}_{2} \text { and DP } \\
\text { (weighted and } \\
\text { not weighted) }\end{array}$ & $\begin{array}{c}\text { Anti-spurious } \\
\text { Filter }\end{array}$ & $\begin{array}{c}\text { Anti-Aliasing } \\
\text { Filter }\end{array}$ & Trasversal \\
\hline
\end{tabular}

The Table 1 highlights that the images belonging to the others categories have been used to refine and optimize the textural feature extraction methodology. Moreover, it shows that two different kinds of pre-processing filters have been applied on the related source image with the aim to normalize the original gray levels. The mentioned filters do not alter the quality of the original source image, but they are only used to improve the few image zones affected from lack of information (i.e. localized noise).

The two screenshots shown in Figure 2 point out the segmentation process of the EMI application on two encaphalic NMR images. In particular, the first screenshot (left) shows an image in which the three layers related to basic objects are found (a). The second one (right) highlights the recognition of four layers 

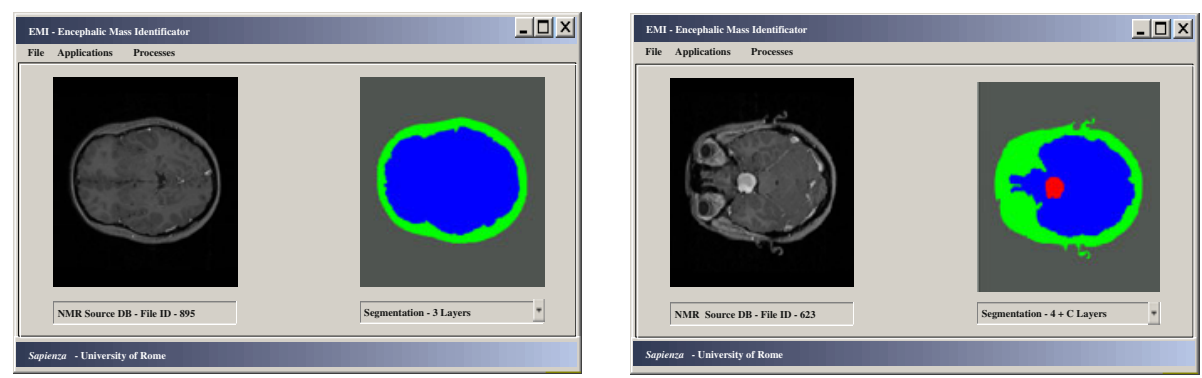

Fig. 2. EMI Application: NMR encephalic segmentation

where it is also identified an abnormal mass classified as craniopharyngioma pathology (a, b and c).

The image analysis process of the EMI application has been based on the following Craniopharyngioma Mathematical Model (CPH-MM):

$$
\begin{aligned}
& \{C P H-M M\}=(((25 \leq C r(1) \leq 145) \vee(198 \leq C r(1) \leq 255)) \wedge \\
& ((33 \leq C r(2) \leq 180) \vee(220 \leq C r(2) \leq 250)) \wedge((23 \leq C r(2) \leq 109) \vee \\
& (148 \leq C r(3) \leq 213))) \wedge(((70 \leq D e(1) \leq 112) \vee(160 \leq D e(1) \leq 200)) \wedge \\
& ((34 \leq D e(2) \leq 57) \vee(100 \leq D e(2) \leq 127)) \wedge((110 \leq D e(3) \leq 200) \vee \\
& (220 \leq D e(3) \leq 255)))
\end{aligned}
$$

Actually, the EMI application needs to take into account also the Abnormal Mass Mathematical Model (AB-MM) which primary support the general abnormal mass detection (see [2]). Subsequently, the application, only on the abnormal mass, can apply the $\mathrm{CPH}-\mathrm{MM}$ with the aim to recognize it as belonging to the craniopharyngioma pathology.

\subsection{Mathematical Reconstruction}

Observe that the MMs, on one side, define the formalized mathematical classes by which to represent the basic objects. On the other hand, they support a first step inside the 3D reconstruction and rendering environment, in fact the provided classes have an exhaustive informative content. A skilled user can already exploit the mathematical classes on different anatomic scanning plans to inference complex information, but one of our next steps is to implement a visual 3D rendering engine.

\section{Experimental Results}

This section summarizes the experimental results regarding the total body NMR image analysis, related to the more advanced current case studies on the following medical domains: brain, heart, liver, bony structure. In order to explain the 
experimental session, the following three general tasks have been selected according to the present medical image analysis process: task 1: layout segmentation, task 2: abnormal mass or lesion detection, task 3: textural characterization.

The Table 2 shows the experimental session which has been subdivided in two different phases. The first, regarding the NMR image recognition, has concerned the selection of patients (455) by which to define the set of training images (1850) to build the four basic MMs. The second, regarding the NMR image classification and segmentation, has concerned the selection of patients (615) by which to obtain a set of images (2565) to test each MM on the mentioned tasks.

Table 2. Main Case Studies: Training and Source DB

\begin{tabular}{|c|c|c|c|c|c|c|c|c|}
\hline \multirow{2}{*}{$\begin{array}{l}\text { Medical } \\
\text { Domain }\end{array}$} & \multirow{2}{*}{ Patients } & \multicolumn{3}{|c|}{ Training DB Images } & \multicolumn{3}{|c|}{ Source DB Images } & \multirow{2}{*}{$\begin{array}{l}\text { Partial } \\
\text { Images }\end{array}$} \\
\hline & & Task 1 & Task 2 & Task 3 & Task 1 & Task 2 & Task 3 & \\
\hline Brain & $270+385$ & 450 & 680 & 60 & 975 & 775 & 40 & 2980 \\
\hline Heart & $75+100$ & 150 & 80 & 35 & 170 & 100 & 25 & 560 \\
\hline Liver & $60+80$ & 70 & 100 & 30 & 135 & 90 & 20 & 445 \\
\hline $\begin{array}{c}\text { Bony } \\
\text { Structure }\end{array}$ & $50+50$ & 70 & 85 & 40 & 100 & 100 & 35 & 430 \\
\hline $\begin{array}{c}\text { Total } \\
\text { Images }\end{array}$ & $455+615$ & 740 & 945 & 165 & 1380 & 1065 & 120 & 4415 \\
\hline
\end{tabular}

Actually, the best qualitative results (more than $90 \%$ of success rate) comes from the brain MM which has a large amount of training and test images. Moreover, it has been our historical first study case. Also the remaining three models have an high success rate (between the $65 \%$ and $80 \%$ ), but each one needs of a more wide experimental session. In fact, the accuracy of the MM is strongly tied to the amount of training images used to refine the mathematical classes.

\section{Conclusions}

This paper describes the main aspects of our developed T-CAD Framework, which exploits the textural information that covers organs and tissues, represented within the NMR total body images, to perform different activities related to the medical image processing field.

The skilled user, once established both the contextual medical domain (e.g. encephalic analysis) and the specific task (e.g. craniopharyngioma recognition) can build the related MM (i.e. the set of suitable mathematical classes) able to describe the basic objects (i.e. cerebral tissue, rest of the image, abnormal mass, craniopharyngioma and background) useful to the achievement of the same task just mentioned (i.e. craniopharyngioma recognition on any compatible image dataset). In order to achieve the task, the framework allows user to create a suitable application based on the related MM. Observe that every MM is defined once and for all. At the moment, our main work is to refine the MM of the 
following organs and tissues: brain, heart, liver, bony structure. Another our goal is the development of a rendering engine to visualize a readable and interactive $3 \mathrm{D}$ objects reconstruction.

\section{References}

1. Avola, D., Cinque, L.: Encephalic NMR Image Analysis by Textural Interpretation. In: Avanzi, R.M., Keliher, L., Sica, F. (eds.) Proceedings of the 2008 ACM Symposium on Applied Computing, SAC 2008. LNCS, March 16-20, pp. 1338-1342. ACM Press, New York (2009)

2. Avola, D., Cinque, L.: Encephalic NMR Tumor Diversification by Textural Interpretation. In: Foggia, P., Sansone, C., Vento, M. (eds.) ICIAP 2009. LNCS, vol. 5716, pp. 394-403. Springer, Heidelberg (2009)

3. Avola, D., Cinque, L., Di Girolamo, M.: Texture Based Approches to Support Medical Image Analysis. Internal Technical Report in Medical Image Proessing. DSI - Sapienza University of Rome, ITR-MIP 2010, Int. Res. on Medical Imaging (2010)

4. Zhou, Y., Bai, J.: Atlas-Based Fuzzy Connectedness Segmentation and Intensity Nonuniformity Correction Applied to Brain MRI. IEEE Trans. on Biomed. Eng. Spons. by IEEE Eng. in Med. and Bio. Soc. 54(1), 122-129 (2007)

5. Li, Q., Shi, Z.: Texture Image Retrieval Using Compact Texton Co-Occurrence Matrix Descriptor. In: Proceedings of the 11th ACM International Conference on Multimedia Information Retrieval, MIR 2010, March 29-31, pp. 83-90. ACM Press, Philadelphia (2010)

6. Histace, A., Matuszewski, B., Zhang, Y.: Segmentation of Myocardial Boundaries in Tagged Cardiac MRI Using Active Contours: A Gradient-Based Approach Integrating Texture Analysis. International Journal of Biomedical Imaging, IJBI 2009, 1-8 (2009)

7. Niranjan, J., Michael, B.: Non-Parametric Mixture Model Based Evolution of Level Sets and Application to Medical Image. International Journal of Computer Vision, IJCV 2010 1(88), 52-68 (2010)

8. Wu, J., Poehlman, S., Noseworthy, M.D., Kamath, M.V.: Texture Feature Based Automated Seeded Region Growing in Abdominal MRI Segmentation. Journal in Biomed. Sc. and Eng., JBiSE 2009 2, 1-8 (2009)

9. Schmid, J., Kim, J., Magnenat-Thalmann, N.: Robust Statistical Shape Models for MRI Bone Segmentation in Presence of Small Field of View. International Journal of Medical Image Analysis, IJMIA 2011 15(1), 155-168 (2011)

10. Tesar, L., Smutek, D., Shimizu, A., Kobatake, H.: Medical Image Segmentation Using Co-Occurrence Matrix Based Texture Features Calculated on Weighted Region. In: Proceedings of the 3rd Conference on IASTED International Conference: Advances in Computer Science and Technology, ACST 2007, pp. 243-248. ACTA Press, Phuket (2007)

11. Heeger, D.J., Bergen, J.R.: Pyramid-Based Texture Analysis/Synthesis. In: Proceeding of 22nd Annual Conference on Computer Graphics and Interactive Techniques, SIGGRAPH 1995, pp. 229-238. ACM Press, NY (1995) 